\title{
Aufsätze
}

Katharina Hanel und Stefan Marschall

\section{Die Nutzung kollaborativer Online-Plattformen durch Parteien: „Top down“ oder „bottom up“?}

\section{Kurzfassung}

Angesichts ihrer „Linkage“-Probleme suchen und testen Parteien neue Wege der Kommunikation, zum Beispiel den Einsatz kollaborativer Online-Plattformen. Durch die Verwendung solcher Internet-Tools können sich im Sinne der Medialisierungsthese die Strukturen der Willensbildung und Entscheidungsfindung innerhalb der Parteien verändern. Entweder kommt es mittels „top down“-Prozessen zu einer Stärkung der Parteiführung oder in Form einer „bottom up“-Beteiligung zu der Verlagerung von Macht auf die Ebene der Parteibasis. Die Analyse des Einsatzes einer internetbasierten Beteiligungsplattform im Vorfeld des Parteitags der SPD im Dezember 2011 zeigt zum einen, dass die Verwendung dieses Tools von der Parteiführung in Prozess und Ergebnisverarbeitung weitreichend kontrolliert und dabei die „bottom up“-Funktionslogik dieser Plattform eingeschränkt wurde. Zum anderen werden robuste Formateigenschaften des Tools sichtbar, welche die Instrumentalisierungsmöglichkeiten seitens der Parteiführung deutlich begrenzen. So ist mittelfristig damit zu rechnen, dass der Einsatz solcher Plattformen die innerparteilichen Willensbildungs- und Entscheidungsprozesse zugunsten von Mitgliedern, aber vor allem auch von Personen, die einer Partei nahestehen ohne jedoch Mitglied zu sein, verändern wird. 


\section{Inhalt}

1. Einleitung

2. Theoretischer Rahmen und Forschungsstand: „Party Change“ durch „Internetisierung“"

3. Fallstudie: onlineantrag.spd.de

a) Entstehung und Umsetzung

b) Nutzung

c) Verarbeitung

d) Verabschiedung 20

4. Diskussion 23

5. Fazit und Perspektiven 25

\section{Einleitung ${ }^{1}$}

Die traditionelle Funktion von Parteien, als intermediäre Akteure zwischen der gesellschaftlichen Basis und dem politisch-administrativen System zu wirken, steht, folgt man aktuellen Diagnosen, vor einer krisenhaften Belastungsprobe: Trotz ihrer starken Rolle im politischen Entscheidungssystem haben die gesellschaftliche Verankerung der Parteien sowie ihre Fähigkeit, als „Sprachrohre“ (Leibholz 1967: 76) der Bürger zu fungieren, abgenommen (von Alemann 2010; Gehne/Spier 2010). Da die Parteien als die zentralen Scharniere der liberalen parlamentarischen Demokratie gelten, wäre eine „Krise der Parteiendemokratie“ zugleich auch eine substanzielle „Krise politischer Repräsentation“ (Linden/Thaa 2011).

Zwar ist die Diagnose von einer Parteienkrise weder neu noch empirisch unumstritten (von Beyme 2000); der ,decline of parties"-Diskurs findet bereits seit Jahrzehnten statt (Immerfall 1994). Ob überhaupt von einer „Krise“ im Sinne eines sich abzeichnenden Systemwechsels die Rede sein kann, ist fraglich. Bislang hat sich die bundesdeutsche Parteiendemokratie als vergleichsweise „krisenresistent“ erwiesen. Dennoch verweisen Indikatoren darauf, dass die Parteien vor erheblichen Herausforderungen stehen, die nicht geringer geworden sind und die den Kern ihrer intermediären Funktion in liberalen repräsentativen Demokratien berühren. Denn Parteien müssen mit einem doppelten „Linkage“-Problem umgehen (Poguntke 2000), welches zum einen ihre Transmissionsfunktion zwischen gesellschaftlicher Basis und politisch-administrativem System, zum anderen die innerparteiliche Wil-

1 Für hilfreiche Anmerkungen danken wir den anonymen Gutachtern/-innen. 
lensbildung und Entscheidungsfindung betrifft. Die Vermittlungsprobleme zwischen Bürgern und staatlichem Entscheidungssystem werden durch einen seit geraumer Zeit greifenden Wandel der gesellschaftlichen Basis mitverursacht: Infolge von Prozessen der Individualisierung und Milieuauflösung haben sich die Strukturen der Gesellschaft ausdifferenziert und heterogenisiert (Gabriel/Falter/Rattinger 2005). Die Parteien sehen sich einem komplexeren Präferenzsystem sowie neuen und vielschichtigen Kommunikationsansprüchen gegenüber (Weiß 2009).

Diese Entwicklungen berühren auch die internen Linkages der Parteien, die Beziehungen zwischen Parteibasis und Parteieliten: Das traditionelle Konzept der Mitgliederpartei steht infrage. Zum einen sind die Mitgliederzahlen in den Parteien rückläufig - ein Trend, der sich in zahlreichen westlichen Demokratien abzeichnet (van Biezen/Mair/Poguntke 2012). Zum anderen leidet die innerparteiliche Demokratie unter einer geringen Beteiligungsbereitschaft der Mitglieder (Spier 2011).

Die Parteien haben in der Bevölkerung an Vertrauen und Zustimmung verloren; dieses Phänomen erstreckt sich nicht nur auf die Regierungsparteien, sondern auch auf die Oppositionsparteien (Wiesendahl 2012). ${ }^{2}$ In diesen Vertrauensproblemen wird ein zentraler Grund gesehen, warum jüngst die Piratenpartei Wahlerfolge in Deutschland hat einfahren können; die „Piraten“ sind von einem beachtlichen Anteil der Wähler gewählt worden, nicht (nur) weil sie ein mobilisierendes Thema entdeckt und besetzt haben, sondern vor allem, weil sie mit dem Nimbus einer „AntiparteienPartei“ aufwarten konnten. $^{3}$

Zum alternativen Angebot der „Piraten“ gehört auch eine neue Art der Nutzung von Medientechniken für die Organisation innerparteilicher Meinungsbildung (Vogelmann 2012), welche neue Linkage-Strukturen zwischen Mitgliedern und Entscheidungseliten konstituiert. Besondere Aufmerksamkeit hat dabei unter dem Rubrum „LiquidFeedback“ die Verwendung einer internetbasierten Beteiligungsplattform gefunden, die eine kollaborative Mitwirkung der Mitglieder in der innerparteilichen Willensbildung und Entscheidungsfindung ermöglicht (Bieber 2011 b; Roleff 2012).

Ähnliche internetbasierte Beteiligungsplattformen sind in den vergangenen Jahren in unterschiedlichen politischen Kontexten eingesetzt worden, beispielsweise bei der Erstellung sogenannter „Bürgerhaushalte“ (Lübcke/Lührs 2008; Märker/ Wehner 2008). Im parlamentarischen Bereich macht die Enquete-Kommission „In-

2 Vergleiche zum Beispiel Statista GmbH, http:/de.statista.com/statistik/daten/studie/153820/umfra ge/allgemeines-vertrauen-in-die-parteien/ (Stand: 21.5.2012).

3 Vergleiche Analysen der Forschungsgruppe Wahlen 2012: Politbarometer April 2012 und Wahlanalyse Nordrhein-Westfalen, www.forschungsgruppe.de/Aktuelles/Politbarometer/ bzw. www.forschungsgruppe.de/Aktuelles/Wahl_NRW/ (Stand: 14.5.2012). 
ternet und digitale Gesellschaft" von einer kollaborativen Online-Plattform Gebrauch. ${ }^{4}$ Und bei den Parteien ist es nicht nur die „Piraten“, sondern es sind auch etablierte Parteien, bei denen der Einsatz solcher Instrumente praktiziert oder angedacht wird. ${ }^{5}$

Im Folgenden steht die Frage im Mittelpunkt, unter welchen Voraussetzungen, in welcher Form und mit welchen Konsequenzen solche Internet-Tools von Parteien eingesetzt werden. Die Fragestellung wird eingebettet in die Debatte um den medien- und technikbedingten Wandel von Parteien (,,party change") und seine Konsequenzen für die dargestellten Linkage-Probleme.

Der Beitrag ist wie folgt aufgebaut: In einem ersten Abschnitt wird skizziert, wie Veränderungen des medialen Systems und von Medientechniken zu einem Wandel der Parteien als Organisationen führen können. Am Ende der Darstellung der Forschungsdebatte werden theoretisch und empirisch fundierte Erwartungen darüber abgeleitet, welche Rolle internetbasierte Partizipationsverfahren in Parteien spielen könnten und mit welchen Konsequenzen ihr Einsatz verbunden sein müsste. Als empirisches Fallbeispiel wird die internetbasierte kollaborative Entwicklung eines Antragskapitels für den Bundesparteitag der SPD im Dezember 2011 herangezogen und in Form einer Fallstudie ausgewertet. Die Ergebnisse der Untersuchung werden an die Debatte um den internet-bedingten ,party change“ zurückgebunden. Sie sollen und können generelle Hinweise auf die Potenziale und Grenzen solcher Instrumente für die innerparteiliche Willensbildung und Entscheidungsfindung geben sowie eine Einschätzung darüber erlauben, ob und inwieweit Online-Kommunikation zur Lösung der Linkage-Probleme beitragen kann.

4 Vergleiche Enquete-Kommission „Internet und digitale Gesellschaft“, www.enquetebeteiligung.de/ (Stand: 21.5.2012).

5 Vergleiche FDP: Chancen für morgen (www.fdp.de/Chancen-fuer-morgen/1053c11794i1p251/index.html), SPD-Bundestagsfraktion: Zukunftsdialog (www.zukunftsdialog.spdfraktion.de/), Die Linke: Programmdebatte (www.dielinke.adhocracy.de/instance/dielinke) (alle Stand: 23.5.2012) sowie FDP (Bayern): New Democracy (https://www.newdemocracy.de/?skin=fdp\&member=1; Stand: 6.8.2012). 


\section{Theoretischer Rahmen und Forschungsstand: „Party Change“ durch „In- ternetisierung“"}

Angesichts der Stabilität der Parteiendemokratie trotz immer wieder verkündeter „Krisen“ hat sich die Parteienforschung auf den Prozess des Wandels von Parteien konzentriert (,party change“). ${ }^{6}$ Als „lernende“ Institutionen (Bandelow 1999) haben Parteien auf die Veränderungen ihrer Umwelt mit Veränderungen ihrer Strukturen reagiert (Wiesendahl 2010).

Als Resultat des „,party change“ wird die Entstehung eines neuen Parteityps beobachtet: Aus den mitgliederbasierten (Volks-)Parteien seien ,,professionalisierte Wählerparteien“ (von Beyme 2000), „moderne Kaderparteien“" (Koole 1996) oder „Netzwerkparteien“ (Heidar/Saglie 2003) geworden. Gemeinsam ist diesen Typisierungen die Unterstellung, dass die innerparteilichen Eliten durch ein professionelles Management an Steuerungspotenzialen hinzugewonnen haben. In eine ähnliche Richtung argumentiert die Kartellparteithese (Katz/Mair 1995), die besagt, dass sich die (etablierten) Parteien(-eliten) abspracheförmig miteinander verbunden und gegen das Aufkommen neuer Parteien abgeschottet haben. In der ,,party change“-Forschung ist überdies von der Etablierung von „Medienkommunikationsparteien“ (Jun 2004) die Rede: Die Parteieliten hätten mithilfe der Massenmedien ihre Entscheidungsmacht ausgebaut und verwenden massenmediale Kanäle auch für die parteiinterne Kommunikation, welche damit Funktionen der früheren Parteipresse übernommen haben (von Alemann 1997).

„Party change“-Konzepte, die die organisatorische Professionalisierung in den Mittelpunkt stellen, treffen sich in der Vermutung, dass die Parteimitglieder eine nur noch nachgeordnete Rolle spielen; primäre Ziele von Parteien wie ,vote seeking“ und „office seeking“ (Müller/Strøm 1999) können unter Umgehung oder zumindest Vernachlässigung der Mitglieder verfolgt werden. Die intermediären und mobilisierenden Aufgaben der Parteibasis werden durch ein professionalisiertes Kommunikationsmanagement der Parteiführung ersetzt oder zumindest ergänzt. Infolge tendiert die Kommunikation mit den Mitgliedern für die Parteieliten zu einer zweiten Ebene der Außenkommunikation zu werden: Mitglieder werden gleichermaßen wie die Nichtmitglieder über die Massenmedien angesprochen. Ob eine solche Kommunikationsstrategie den „Abgesang der Mitgliederpartei“ einläutet, wird kontrovers diskutiert; fest steht jedoch, dass sich die funktionale Rolle der Parteimitglieder im Wandel befindet. ${ }^{7}$

6 Siehe hierzu etwa von Beyme 2000, Detterbeck 2002 oder Wiesendahl/Jun/Niedermayer 2009.

7 Vergleiche Jun 2009, Klein/Alemann/Spier 2011, Wiesendahl 2009 und Detterbeck 2005. 
Allein der Begriff der Medienkommunikationspartei macht deutlich, dass ,,party change" mit den Veränderungen im Mediensystem verbunden ist. ${ }^{8}$ Wie andere Umweltveränderungen wirkt auch der „Medienwandel“ (Schulz 2011) auf die Parteien ein; diese passen sich an oder werden angepasst; jedenfalls verändern sie sich infolge des Wandels der Medien - ein Mechanismus, den man mit dem Begriff der „Medialisierung“9 fasst. „Medialisierung“ wird in der Kommunikations- und Politikwissenschaft als ein vielschichtiges analytisches Konzept diskutiert, sodass sowohl im Hinblick auf die Bedeutung des Begriffs selbst als auch im Hinblick auf die Reichweite der Medialisierung von Politik keine eindeutigen Annahmen und Befunde vorliegen (Donges 2005). ${ }^{10}$

Für die Parteien ist der Medienwandel Risiko und Chance zugleich. Zum einen stellen Veränderungen der medialen Umwelt eine Herausforderung an die Vermittlungsleistungen der Parteien dar. Zum anderen können die Parteien Medien nutzen, um Machtpotenziale auszubauen oder wiederzugewinnen, indem sie neue Medienstrukturen instrumentalisieren. Medialisierung reflektiert sich nicht nur in der Außenkommunikation der Parteien, sondern schlägt sich auch in den parteiinternen Prozessen der Politikvermittlung nieder (Donges 2008; Jun 2004), zum Beispiel in der bereits erwähnten Verwendung von Massenmedien für die innerparteiliche Kommunikation.

Ein substanzieller Teil des auf die Parteien wirkenden Medienwandels der vergangenen Jahre stellen die Etablierung und Konsolidierung des Internet als Plattform für politische Kommunikation und Information dar (Emmer/Vowe/Wolling 2011). Empirische Untersuchungen machen eine generelle Bereitschaft bei den Parteieliten aus, neue Informations- und Kommunikationstechnologien für ihr nach innen und außen gerichtetes Kommunikationsmanagement zu nutzen (Bieber 1999; Gibson/Nixon/Ward 2003).

Welche Implikationen die „Internetisierung“ der Politik (Sarcinelli/Wissel 1996) auf die interne Organisation von Parteien hat, ist dabei kontrovers diskutiert worden: Zum einen stand bereits frühzeitig die Hoffnung im Raum, Internet-Kommunikation erlaube aufgrund ihrer reziproken und teilnahmeoffenen Strukturen, Willensbildungs- und Entscheidungsprozesse partizipativ auszuweiten (,,bottom up“) und die

8 So werden beispielsweise als Entstehungsbedingungen des Typs „Medienkommunikationspartei“ neben Veränderungen der politischen und gesellschaftlichen Kontextbedingungen von Parteien explizit Veränderungen der medialen Umwelt angesehen (Jun 2009).

9 Vergleiche Mazzoleni/Schulz 1999, Donges 2008 sowie Schulz 2011.

10 Eine systematische Auseinandersetzung und Einordnung des Begriffs liefern Donges 2008 und Schade 2004. Medialisierung sollte, so schlägt es Schade (2004) vor, von Medialisierungsfolgen, Medialisierungslogik und Medialisierungsstrategie unterschieden werden. 
Mitglieder von Parteien, aber auch Nichtmitglieder einzubeziehen (im Weiteren: „Partizipationsthese“, vergleiche Meckel 2008, Leggewie/Bieber 2004).

Dem stand und steht die skeptische Erwartung gegenüber, die vorherrschenden „top down“-Strukturen innerhalb der Parteien würden durch das Internet nicht aufgehoben, sondern verstärkt, weil die Parteieliten die neuen Kommunikations- und Informationstechnologien strategisch nutzten, um sich zusätzliche Steuerungsvorteile zu verschaffen (im Weiteren: „Instrumentalisierungsthese“, vergleiche Sarcinelli 2011, Wiesendahl 2002).

Diese Debatte ist durch das Aufkommen des Web 2.0 wiederbelebt worden diesmal deutlich zugunsten der „Partizipationsthese“, da sich das „Social Web“ gerade durch seine vernetzten und antihierarchisch-egalitären Eigenschaften auszeichnet (Busemann/Gscheidle 2011), also eine „,bottom up“-Öffnung von Willensbildungs- und Entscheidungsprozessen technisch erleichtern könnte (Bieber $2011 \mathrm{~b})$.

Mittlerweile sind pauschale, auf das Internet als „Medium“ gerichtete Erwartungen einer differenzierteren Sichtweise gewichen. In der Kommunikations- und Medienwissenschaft wird das Internet nicht (mehr) als monolithisches Medium begriffen, sondern als eine Vielzahl unterschiedlicher „Medien zweiter Ordnung“ konzeptualisiert, die auf einer gemeinsamen technischen Basis, einem „Medium erster Ordnung", angesiedelt sind und sich in ihren kommunikativen Merkmalen erheblich unterscheiden (Beck 2010). Folgt man einem solch differenzierten Ansatz, können die Erwartungen an ,das Internet“ und seine Effekte auf politische Akteure und Prozesse nur „toolspezifisch“ formuliert und untersucht werden.

Dieser Ansatz greift mutatis mutandis auch für das Web 2.0. Beim „Social Web“ handelt es sich um einen Sammelbegriff für höchst unterschiedliche Anwendungen (Ebersbach/Glaser/Heigl 2011), die jede für sich in ihrer Auswirkung auf politische Prozesse und politische Akteure, zum Beispiel die Parteien, analysiert werden sollten.

$\mathrm{Zu}$ den Web 2.0-Tools, die in jüngster Zeit Einsatz gefunden haben und mittlerweile auch von den Parteien eingesetzt worden sind, werden auch die kollaborativen Plattformen gezählt, die auf dem Wiki-Prinzip und der Wiki-Technik beruhen (Schmidt 2011). Diese zeichnen sich dadurch aus, dass sie den Nutzern die technische Grundlage geben, Texte und Dokumente zu entwickeln, zu kommentieren und zu ändern. Aufgrund ihrer Formatstrukturen bieten diese interaktiven Beteiligungsplattformen die Möglichkeit, viele Personen gleichzeitig, gleichberechtigt, sachbezogen und transparent an der Formulierung, Diskussion und Verabschiedung von Texten teilnehmen zu lassen (Ebersbach/Glaser/Heigl 2011). 
In jüngster Zeit sind Software-Varianten wie „LiquidFeedback“ oder „Adhocracy" diskutiert und angewendet worden, ${ }^{11}$ die Funktionen der gemeinsamen Textbearbeitung durch Abstimmungsmechanismen ergänzen. ${ }^{12}$ So beschreiben beispielsweise die Entwickler von Adhocracy ihre Software wie folgt: „Adhocracy is a policy drafting tool for distributed groups. It allows members of organizations or the public to compose or vote documents that represent the policy of the group". ${ }^{13}$ Adhocracy ermöglicht also das gemeinsame Er- und Bearbeiten von Texten sowie die Herbeiführung von Meinungsbildern und Präferenzabstimmungen innerhalb einer Gruppe.

Die Wahlerfolge der Piratenpartei haben ein Schlaglicht auf die Nutzung solcher Plattformen durch Parteien geworfen, da die „Piraten“ wie bereits erwähnt für einen Großteil der internen Willensbildungs- und Entscheidungsprozesse (z. B. für die Erstellung von programmatischen Texten) auf das Web 2.0 zurückgreifen (Bieber 2012; Lewitzki 2011). Ihre Erfolge werden nicht zuletzt auch dieser neuen parteiinternen, partizipativ ausgerichteten Kommunikationstechnik zugeschrieben (Niedermayer 2010).

Während die Piratenpartei mit der Nutzung von internetbasierten Beteiligungsinstrumenten „groß geworden“ ist, würde die Verwendung solcher Tools für etablierte Parteien mit Änderungen ihrer bestehenden Willensbildungs- und Entscheidungsprozesse einhergehen. Deswegen ist im Sinne der Medialisierungsthese der Einsatz von kollaborativen Plattformen in einer etablierten Partei heuristisch besonders aufschlussreich, weil es dort gegebenenfalls möglich ist, Art und Ausmaß solch medieninduzierter Veränderungen in den Strukturen und Ergebnissen der Meinungsbildungs- und Entscheidungsprozesse zu beobachten.

11 Die Plattformsoftware „Adhocracy“ ist unter anderem für die Beteiligung von Bürgern in der bereits angesprochenen Enquete-Kommission „Internet und digitale Gesellschaft“ des Bundestages, für das SPD „Projekt Zukunft“ sowie für eine elektronische Programmdebatte der Partei Die Linke verwendet worden.

12 Innerhalb der Piratenpartei sowie aus Sicht der Entwickler der beiden angesprochenen SoftwareVarianten, Liquid Democracy e. V. („Adhocracy“) und Interaktive Demokratie e. V. („LiquidFeedback"), werden die Plattformen als praktische Umsetzung einer sogenannten ,Liquid Democracy" konzipiert, einem (noch unfertigen) Konzept, das die Vorteile direkter und repräsentativer Demokratiemodelle verbinden soll. Siehe Liquid Democracy e. V., http://liqd.net/schwerpunkte/ theoretische-grundlagen/, und Interaktive Demokratie e. V., http://liquidfeedback.org/lqfb/lqfb-de/ (Stand: 6.8.2012).

Die Unterschiede zwischen den theoretischen Konzepten der beiden Vereine, die sich zum Teil auch in der Software niederschlagen, können an dieser Stelle nicht weiter diskutiert werden. Siehe dazu u. a.: Interaktive Demokratie e. V., http://liquidfeedback.org/2011/08/17/5-jahre-liquid-dem ocracy-in-deutschland/ (Stand: 6.8.2012).

13 Adhocracy Wiki, www.trac.adhocracy.de (Stand: 21.5.2012). 
Welche konkreten Erwartungen lassen sich an den Einsatz einer kollaborativen Online-Plattform durch eine etablierte Partei formulieren? Darüber, ob und in welcher Form solche Plattformen eingesetzt werden, können die Parteien selbst entscheiden. Dies unterscheidet den Einsatz von Beteiligungsplattformen von der Nutzung anderer Web 2.0-Angebote wie Facebook oder Twitter, bei denen die Parteien die Formatbedingungen der Kommunikation nicht steuern können, sondern diese von den externen Anbietern bestimmt werden. Die Entscheidung über das „ob“ und „wie“ des Einsatzes von gestaltbaren Systemen liegt bei der jeweiligen Parteiführung; hier müsste die beobachtete Professionalisierung und Zentralisierung der Online-Kommunikation innerhalb der Parteien greifen (Albers 2009).

Die Forschung hat sich über Jahrzehnte mit „Oligarchisierungsprozessen“ in Parteien auseinandergesetzt und herausgearbeitet, dass sich Parteiführungen (,party in central office“) gegenüber der innerparteilichen Basis (,party on the ground“) abgekoppelt, verselbstständigt und professionalisiert haben (Katz/Mair 2002). Dies spricht dafür, dass die Parteiführungen kein Interesse an der Öffnung und Erweiterung von bestehenden Willensbildungs- und Entscheidungsprozessen haben dürften, gingen ihnen dabei Machtpotenziale verloren. Deswegen steht zu vermuten, dass Parteiführungen, solange sie über den innerparteilichen Einsatz von Web 2.0Tools entscheiden können, ihre Verwendung nur soweit zulassen, wie es auf der Grundlage einer Kosten-Nutzen-Kalkulation naheliegt. Chadwick (2009) identifiziert zahlreiche Faktoren, die in einer solchen Kalkulation berücksichtigt werden und sich auf den Einsatz internetbasierter Anwendungen im politischen Kontext auswirken können. Dazu gehört neben zeitlichen, finanziellen und personellen Ressourcen sowie - vor dem Hintergrund partizipativer Asymmetrien - Zweifeln an der Repräsentativität der Teilnehmenden und der geäußerten Meinungen explizit auch die Angst vor einem Kontroll- und Steuerungsverlust auf Seiten der politischen Eliten.

Jenseits der Gewährleistung der Prozesssteuerung legt eine „rational choice“Perspektive die Sicherung der Ergebnisverarbeitung nahe. Es ist davon auszugehen, dass die Parteiführung bei der Verarbeitung des Prozessergebnisses keine Automatismen zulässt, sondern Korrektur- und Filtermechanismen einbaut. ${ }^{14}$ Dies erscheint alleine deswegen naheliegend, als dass bei Online-Anwendungen immer wieder die Gefahr der Manipulation von externer Seite diskutiert wird.

Sowohl aus Sicht der Medialisierungsthese als auch aus einer organisationstheoretischen Perspektive heraus lässt sich argumentieren, dass bestehende institutionelle Strukturen, Akteurskonstellationen sowie die Eigenlogik des Anwendungs-

14 Siehe Jackson/Lilleker 2009, Stromer-Galley 2000 sowie Tedesco 2007. 
kontextes auf die Ausgestaltung und die Auswirkung von Beteiligungsverfahren in Parteien Einfluss nehmen. ${ }^{15}$ Mithilfe eines Fallbeispiels sollen folgende zwei, nach Phasen des Prozesses unterschiedene Thesen überprüft werden, die beide auf der gleichen Grundvermutung der Festigkeit bestehender Parteihierarchien und institutioneller Strukturen basieren:

These 1: Während der Online-Phase wird aufgrund der Robustheit bestehender Willensbildungs- und Entscheidungsstrukturen die Parteiführung beim innerparteilichen Einsatz einer Partizipationsplattform die Prozesssteuerung behalten.

These 2: Nach der Online-Phase wird die Parteiführung die Steuerungskompetenz über die Verarbeitung des Ergebnisses nicht aus der Hand geben.

Jede beobachtbare Abweichung von diesen Erwartungen könnte dahingehend verstanden werden, dass die verwendeten Tools eine robuste Eigenlogik haben, welche die Instrumentalisierungsmöglichkeiten einschränkt. Die Eigenlogik des Mediums stieße dann auf die Eigenlogik der Organisation.

\section{Fallstudie: onlineantrag.spd.de}

Als Gegenstand der Fallstudie dient der Einsatz der kollaborativen Internet-Plattform „onlineantrag.spd.de“ 16 durch die Sozialdemokratische Partei Deutschlands. Vom 4. August bis zum 19. September 2011 hatten Mitglieder der SPD wie auch Nichtmitglieder die Möglichkeit, sich auf einer Online-Plattform an der Formulierung des Kapitels „Arbeit und Wirtschaft in der Digitalen Gesellschaft“" zu beteiligen, das Teil des Leitantrags ,Freiheit, Gerechtigkeit und Solidarität in der Digitalen Gesellschaft" des SPD-Parteivorstands auf dem Bundesparteitag im Dezember 2011 sein sollte.

Die Fallstudie orientiert sich methodisch am Vorgehen anderer Fallstudien, die online-basierte politische Beteiligungsprozesse analysiert und evaluiert haben. ${ }^{17}$ Wie bei Fallstudien üblich, wurde auch bei der vorliegenden Analyse ein Methodenmix aus inhaltsanalytischen Verfahren, teilnehmender Beobachtung und Interviews angewandt (Häder 2010).

Konkret wird wie folgt vorgegangen: Zunächst wird auf die Entstehung und die Umsetzung des Online-Beteiligungsverfahrens eingegangen; dazu ist unter anderem

15 Siehe hierzu Chadwick 2011 und 2009, Wiesendahl 2010, Ward/Gibson 2009, Sarcinelli 2004, Mazzoleni/Schulz 1999.

16 Die Plattform basiert auf der angesprochenen Software „Adhocracy“ des Vereins Liquid Democracy e. V.

17 Beispielsweise Kubicek/Westholm 2010, Lilleker/Pack/Jackson 2010; ausführlich zur Methodendiskussion bei der Evaluation von Online-Beteiligungsprojekten Aichholzer/Westholm 2009, Abelson/Gauvin 2006. 
eine Analyse der Plattformstrukturen im Hinblick auf Partizipations- und Interaktionsoptionen durchgeführt worden (Christman u. a. 2010; Wölk u. a. 2008). Anschließend stellen wir dar, wie die Beteiligungsangebote auf der Plattform tatsächlich genutzt wurden. In einem nächsten Schritt wird nachvollzogen, nach welchen Entscheidungs-/Verfahrensregeln die Beiträge der Teilnehmenden für die Antragsformulierung verarbeitet und zu einem Ergebnis aggregiert wurden. Zusätzlich wird inhaltsanalytisch untersucht (Lamnek 2005; Rössler 2010), welchen Anteil die Online-Diskussion am fertigen Antragskapitel einnimmt und wie sich die Beiträge der Teilnehmenden im Antragskapitel widerspiegeln. Abschließend ist im Rahmen einer teilnehmenden Beobachtung (Häder 2010) erfasst worden, wie der Antrag in die Beschlussverfahren des Parteitags integriert wurde. Die Ergebnisse der Analyse des Beteiligungsverfahrens auf der Online-Plattform sowie der teilnehmenden Beobachtung werden durch Erkenntnisse aus leitfadengestützten Experteninterviews (Blatter/Janning/Wagemann 2007) mit verantwortlichen Projektbeteiligten innerhalb der SPD und Liquid Democracy e. V. ergänzt, die im Rahmen des Parteitags geführt worden sind. ${ }^{18}$ Auf der Grundlage dieser Befunde sollen die beiden im zweiten Abschnitt entwickelten Thesen diskutiert werden.

\section{a) Entstehung und Umsetzung}

Die Initiative des Online-Antrags steht in einem engen Zusammenhang mit der Organisationsreform der SPD, die ein Kernstück des Bundesparteitags im Dezember 2011 darstellte und im Rahmen derer die SPD die Beteiligungsmöglichkeiten für Mitglieder, aber auch Nichtmitglieder auszuweiten plante (Klug 2012). Dieser geplanten Öffnung der Partei wollte man mit dem Online-Antrag auch nach außen ein Gesicht geben. ${ }^{19}$ So beansprucht die Partei für sich, ,jungen Menschen, die sich nicht ortsgebunden in Gliederungen der Partei engagieren können oder wollen, sowie für Menschen, die nur themenbezogen in einer Partei mitarbeiten wollen, erstmalig eine einflussreiche politische Partizipationsmöglichkeit über das Netz" (Bücker 2011: 16) geschaffen zu haben. Beteiligte Akteure innerhalb der Partei waren

18 Es wurden drei Experten befragt. Auf Seiten der SPD-Parteizentrale wurde Tobias Nehren interviewt, der als Mitarbeiter im SPD Newsdesk das Online-Antragsverfahren begleitet hat. Außerdem wurde Daniel Reichert interviewt, der Mitbegründer und Vorstandsvorsitzender des Vereins Liquid Democracy e. V. ist und den Einsatz von „Adhocracy“ für das Online-Antragsverfahren betreute. Zusätzlich konnte ein Interview mit der Community Managerin der Plattform, Eva Breitbach, geführt werden. Die Interviews wurden in digitaler Form aufgenommen und anschließend mit der Software f4 transkribiert und im Hinblick auf wichtige Aussagen zu den Phasen der Fallstudie analysiert.

19 Interview Nehren, SPD. 
als Antragssteller die Medienkommission beim Parteivorstand sowie das SPDNewsdesk des Parteivorstands, ${ }^{20}$ das in der Parteizentrale angesiedelt ist. Wenn also in der Folge von „Parteiführung“ gesprochen wird, sind damit im Sinne der ,party in central office" sowohl Vertreter der politischen Führung als auch Mitarbeiter der Parteizentrale gemeint (Katz/Mair 1993; Bukow 2010).

Die Parteiführung wählte als Gegenstand für das online-basierte Beteiligungsverfahren ein netzpolitisches Thema - gerade auch vor dem Hintergrund der Erfolge der Piratenpartei bei den Wahlen zum Berliner Abgeordnetenhaus. ${ }^{21}$ Vor dem Start des Online-Antragsverfahrens organisierten die Verantwortlichen innerhalb der Partei eine Auftaktveranstaltung, zu der Akteure aus der netzaffinen Berliner Gründerszene eingeladen wurden. So wurde versucht eine für das Antragsthema relevante Zielgruppe für den Prozess zu mobilisieren, von der man sich Expertise und Impulse für dieses für die Partei neue Thema erhoffte. ${ }^{22}$ Darüber hinaus wurden auch die Parteimitglieder über die parteiinternen Kommunikationswege auf spd.de sowie über den Youtube-Kanal der Partei (,SPDvision“) über die Möglichkeit zur Beteiligung am Online-Antragsverfahren informiert.

Technisch umgesetzt wurde die Online-Plattform „onlineantrag.spd.de“auf Basis der bereits angesprochenen Open-Source- und Social-Software Adhocracy, die für den Einsatz in der SPD modifiziert wurde. Die Plattform verfügte in der für das Online-Antragsverfahren verwendeten Version über drei Partizipationsmodi: Vorschläge erstellen und bearbeiten, Kommentieren sowie Abstimmen. Um aktiv am Antragskapitel mitarbeiten zu können, war eine Registrierung auf „onlineantrag.spd.de" erforderlich, allerdings war der Prozess auch für nicht-registrierte Internet-Nutzer während der Online-Phase vollständig sichtbar. Bei der Registrierung konnten die Nutzer wählen, ob sie ihren Klarnamen angeben oder unter einem anonymen Benutzernamen firmieren wollten. Die Teilnehmer hatten außerdem die Möglichkeit, persönliche Angaben sowie ein Foto einzustellen. Die Parteimitgliedschaft wurde nicht abgefragt, ist von manchen Nutzern aber im persönlichen Profil angegeben worden.

Für die Entwicklung von Vorschlägen wurden seitens der Organisatoren sechs Leitfragen zum Themenkomplex ,Arbeit und Wirtschaft in der Digitalen Gesellschaft“ entwickelt und auf der Plattform eingestellt. Über sogenannte „Beobach-

20 Das Newsdesk des SPD-Parteivorstands bezeichnet eine neu geschaffene, eigenständige Redaktion, die das 2010 neu konzeptionierte Partei- und Nachrichtenportal spd.de betreut (Bücker 2011).

21 Interview Nehren, SPD. Siehe auch den Artikel „Piraten im Umfragehoch. Strategie gegen den Freibeuter-Angriff" in der Zeitschrift Stern vom 10.4.2012 (www.stern.de/politik/deutschland/pir aten-im-umfragehoch-strategien-gegen-den-freibeuter-angriff-1811664.html; Stand: 21.5.2012).

22 Interview Nehren, SPD. Siehe auch Pöttgen 2011. 
tungslisten" war es den Teilnehmern möglich, per E-Mail oder Twitter-Mitteilung über aktuelle Entwicklungen bei ausgewählten Vorschlägen oder über Aktivitäten einzelner User informiert zu werden. Direkte Interaktionen zwischen den Nutzern waren in Form von Nachrichten über die Plattform möglich. Zusätzlich wurde für „onlineantrag.spd.de“ zum ersten Mal in einem Adhocracy-basierten Beteiligungsverfahren ein Community Management eingesetzt, um den Prozess auf der Plattform $\mathrm{zu}$ begleiten. ${ }^{23}$ Als Community Managerin wurde eine Partei-Externe beauftragt, die als Ansprechpartnerin auf der Plattform aufgetreten ist. Die Community Managerin war auch nach Abschluss der Onlinephase gemeinsam mit dem verantwortlichen Redakteur des SPD-Newsdesk an der Aggregation und Verarbeitung des Inputs beteiligt.

Das Beteiligungsverfahren verlief in zwei Stufen: In der ersten Phase (04.08.-13.9.2011) konnten die Teilnehmenden die seitens der Organisatoren vorgegebenen Leitfragen zum Themenkomplex des Antragskapitels kommentieren und Vorschläge zur inhaltlichen Positionierung der Partei formulieren. In einer zweiten Phase (13.09.-19.9.2011) fand die Abstimmung über die Vorschläge statt. Die Vorschlagsentwürfe mit den meisten Unterstützern sollten der Beschreibung auf ,onlineantrag.spd.de“" zufolge in die Formulierung des Antrags einbezogen werden.

\section{b) Nutzung ${ }^{24}$}

Im analysierten Zeitraum vom 4.8.2011 bis zum 19.9.2011 haben sich 408 Teilnehmer registriert, ${ }^{25}$ welche die auf der Plattform angebotenen Partizipationsformen der Vorschlagserstellung, Kommentierung und Abstimmung unterschiedlich stark nutzten.

23 Interview Daniel Reichert, Liquid Democracy e. V. Community Manager operieren als Schnittstelle zwischen den Initiatoren von online-basierten Beteiligungsprozessen und den Teilnehmenden auf der Plattform und können einen wichtigen Faktor für den Erfolg online-basierter Beteiligungsprojekte darstellen, da sie die Kommunikation auf der Plattform begleiten und zum Beispiel Fragen und Probleme der Teilnehmenden direkt beheben. Siehe auch Bundesverband Deutscher Community Manager, www.bvcm.org/ (Stand: 22.5.2012).

24 Eine Befragung der Teilnehmenden auf „onlineantrag.spd.de“ war nicht möglich, sodass keine Aussagen über die Teilnehmenden (Parteimitgliedschaft, Soziodemografie, Beteiligungsmotivation usw.) getroffen werden können.

25 Berücksichtigt wurden alle Teilnehmer mit dem Status „Wähler“. So wurden die Teilnehmer auf der Plattform bezeichnet, die nicht zum Organisationsteam gehörten. Die Organisatoren wurden als „Verwalter“ bezeichnet. 


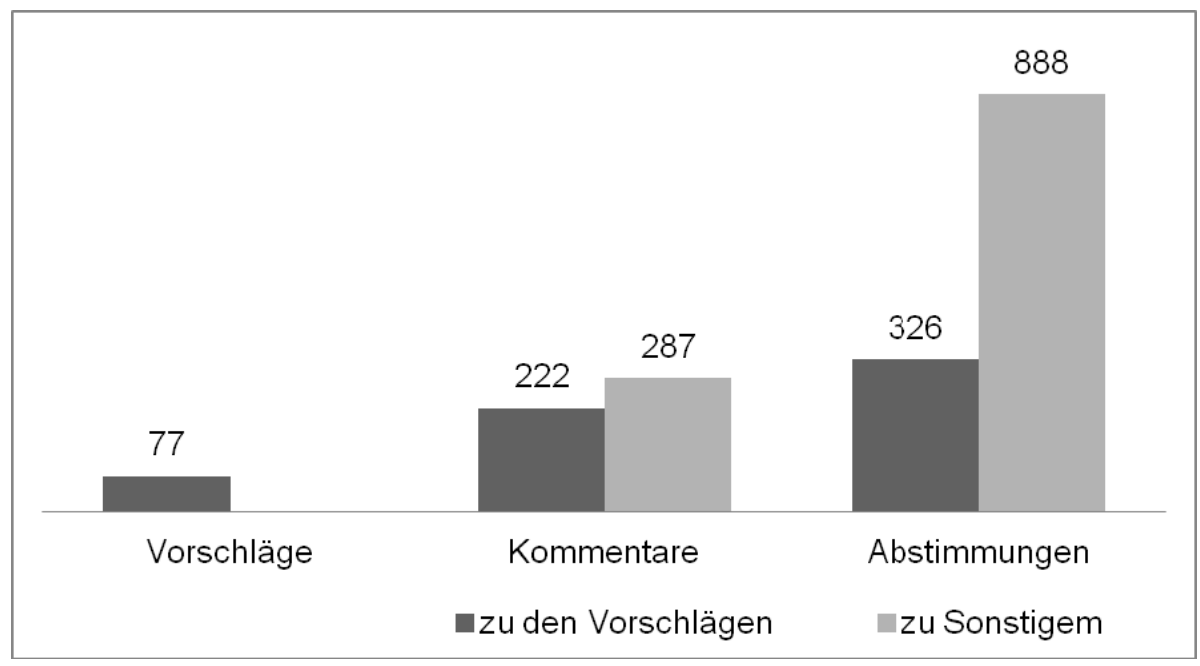

Quelle: www.onlineantrag.spd.de, eigene Darstellung.

Im Untersuchungszeitraum wurden von den Nutzern zu den sechs aufgeworfenen Leitfragen insgesamt 77 Vorschläge erstellt, zu denen wiederum 222 Kommentare abgegeben und 326 Abstimmungen vorgenommen wurden (vgl. Abb. 1). Bezieht man alle Aktivitäten auf der Plattform mit ein, also nicht nur solche, die sich auf die 77 Vorschläge beziehen, sind es insgesamt 509 Kommentare und 1.214 Bewertungen (Abstimmungen). Die Differenz bei den Abstimmungen ergibt sich daraus, dass auch Kommentare bewertet werden konnten. Die zusätzlichen Kommentare stammen aus der Rubrik „Diskussion“, innerhalb derer Fragen zum Prozess usw. diskutiert werden konnten.

Bei der Verteilung dieser Aktivitäten auf die registrierten Teilnehmer fällt auf, dass eine Mehrheit von 66 Prozent (271 Nutzer) überhaupt nicht auf der Plattform aktiv war. Rund ein Drittel (125 Nutzer) hat mindestens eine und maximal zwei Partizipationsformen genutzt, während nur eine kleine Minderheit von drei Prozent (12 Nutzer) von allen drei Beteiligungsformen Gebrauch gemacht hat.

Die Funktion, kollaborativ Vorschläge zu erarbeiten, wurde ebenfalls nur begrenzt genutzt. Die Entscheidung darüber, einen Vorschlag zur gemeinsamen Bearbeitung zu öffnen, oblag denjenigen Nutzern, die diesen erstellt hatten. 77 Prozent der Vorschläge waren erst gar nicht für die gemeinsame Bearbeitung auf der Plattform freigegeben. Die übrigen waren für die gemeinsame Bearbeitung geöffnet, 
allerdings wurden nur vier Vorschläge tatsächlich von mehreren Benutzer weiterentwickelt.

\section{c) Verarbeitung}

Nachdem die Diskussions- und Abstimmungsphase auf der Plattform beendet war, lag die Verarbeitung des online-generierten Inputs in ein abstimmungsfähiges Antragspapier wieder bei den Organisatoren, also dem Parteimanagement unter Mithilfe der Community Managerin. Auf diesen Verarbeitungsprozess hatten die Online-Teilnehmer nur einen geringen Einfluss: Während der Abstimmungsphase konnte die „Community“ zwar die Vorschläge unterstützen. Doch gerade in der darauf folgenden wichtigen Phase zeigte der Prozess Inkonsistenzen: Bevor die Verarbeitung der 77 Vorschläge erfolgte, wurden diese durch das Community Management mit dem Status ,angenommen“ (25 Vorschläge), ,abgelehnt“ (8 Vorschläge) oder ,nicht zum Thema/anderes Thema“ (42 Vorschläge) eingestuft (siehe Tab. 1). Der Status eines jeweiligen Vorschlags war dabei zwar für alle Teilnehmenden in der Übersicht sowie der Einzelansicht der Vorschläge sichtbar, dagegen war nicht transparent, nach welchen Kriterien die Einordnung durch das Community Management erfolgt war. So erhielten sechs Vorschläge trotz Bezugs zum Rahmenthema des Antrags und mehrheitlicher Zustimmung den Status ,abgelehnt“. Dennoch wurde wiederum einer dieser Vorschläge in das Antragskapitel aufgenommen.

Tabelle 1: Einordnung der Vorschläge durch Teilnehmer und Community Management

\begin{tabular}{|c|c|c|c|c|c|}
\hline & & \multicolumn{3}{|c|}{ Abstimmung durch Teilnehmer } & \multirow[b]{2}{*}{$\begin{array}{l}\text { Ge- } \\
\text { samt }\end{array}$} \\
\hline & & $\begin{array}{l}\text { Zustim- } \\
\text { mung }\end{array}$ & $\begin{array}{l}\text { Ableh- } \\
\text { nung }\end{array}$ & $\begin{array}{l}\text { Ausgewo- } \\
\text { gen }\end{array}$ & \\
\hline \multirow{4}{*}{$\begin{array}{l}\text { Status durch Community Manage- } \\
\text { ment }\end{array}$} & „angenommen“ & 24 & 0 & 1 & 25 \\
\hline & ,abgelehnt“ & 6 & 0 & 2 & 8 \\
\hline & „anderes Thema“ & 21 & 5 & 16 & 42 \\
\hline & $\begin{array}{l}\text { kein Status zugewie- } \\
\text { sen }\end{array}$ & 0 & 0 & 2 & 2 \\
\hline \multicolumn{2}{|l|}{ Gesamt } & 51 & 5 & 21 & 77 \\
\hline
\end{tabular}

Quelle: onlineantrag.spd.de, eigene Darstellung.

Insgesamt wird im Antragskapitel auf 19 der 33 thematisch passenden Vorschläge der Online-Diskussion verwiesen. Über diese 19 Vorschläge hinaus hatten zwölf weitere Vorschläge die mehrheitliche Zustimmung der Teilnehmer erhalten, wurden 
aber nicht in das Antragskapitel aufgenommen. Nach welchen Kriterien die 19 Vorschläge ausgewählt worden sind, ist nicht unmittelbar ersichtlich.

Der inhaltsanalytische Vergleich des auf der Plattform erzeugten Inputs mit dem vorgelegten Antragskapitel erbringt weitere aufschlussreiche Befunde. Um zu ermitteln, inwieweit sich die auf der Plattform erarbeiteten Vorschläge tatsächlich im fertigen Antragskapitel wiederfinden, ist zunächst der quantitative Anteil des online-erstellten Inputs an dem im Rahmen des Parteitags zur Abstimmung vorgelegten Antragskapitel ermittelt worden. Demnach sind rund 18 Prozent des Antragskapitels an den Input der Online-Diskussion angelehnt. Die 19 übernommenen Vorschläge finden sich dabei mit unterschiedlich starkem Abstraktionsniveau im Antragskapitel wieder. Während sechs Vorschläge wörtlich übernommen wurden, weisen sieben einen sinngemäßen Bezug und sechs Vorschläge einen sehr abstrakten Bezug zum online erstellten Vorschlag auf; die integrierten Vorschläge wurden also mehrheitlich redaktionell bearbeitet.

Tabelle 2: Ver-/Bearbeitung der als thematisch passend eingestuften Vorschläge

\begin{tabular}{|l|c|c|}
\hline Art der Übernahme in den Antrag & Häufigkeit & Prozente \\
\hline wörtliche Übernahme & 6 & 18,2 \\
\hline sinngemäßer Bezug & 7 & 21,2 \\
\hline sehr abstrakter Bezug & 6 & 18,2 \\
\hline nicht übernommen & 14 & 42,4 \\
\hline gesamt & 33 & 100 \\
\hline
\end{tabular}

Quelle: onlineantrag.spd.de, eigene Darstellung.

\section{d) Verabschiedung}

Nach Abschluss der Online-Phase wurde das Antragskapitel auf „onlineantrag.spd.de“ und auf „spd.de“ veröffentlicht. Damit war der internetbasierte Beteiligungsprozess abgeschlossen. Das Antragskapitel war als Teil des Leitantrags „Freiheit, Gerechtigkeit und Solidarität in der Digitalen Gesellschaft“ des Parteivorstands an die Antragskommission des Bundesparteitags übergeben worden, die den Delegierten die Annahme des Antrags mit einer Änderung empfohlen hatte. ${ }^{26}$ Erarbeitet wurde der Leitantrag von der Medienkommission des Parteivorstands. Aus formalen Gründen fungierte allerdings der Parteivorstand als Antragssteller.

26 Diese betraf allerdings nicht das online erstellte Kapitel. Die Änderung der Antragskommission bezog sich auf Formulierungen im zweiten und vierten Kapitel des Leitantrags, vergleiche Antragsbuch zum Bundesparteitag 2011, S. 410 und 419. 
Somit durchlief der Antrag den im Organisationsstatut der SPD festgelegten Weg eines Antrags an den Bundesparteitag (§ 18 SPD-Organisationsstatut, Stand: Dezember 2011).

Der Leitantrag wurde am zweiten Tag des Parteitags, am Montag den 5. Dezember 2011, gegen 12:55 Uhr im Antragsbereich M (Medien- und Kulturpolitik) durch Björn Böhning, den Leiter des Gesprächskreises Netzpolitik der SPD, eingebracht. Anschließend sprachen Valentina Kerst, Leiterin des Forums Netzpolitik der SPD Köln, Mathias Richel als Vorstandsvorsitzender des Zentrums für digitalen Fortschritt D-64 sowie Matthias Groote (MdEP). Abgeschlossen wurde die Beratung durch die Rede von Alexander Schweitzer, der für die Antragskommission sprach. Gegen ca. 13:15 Uhr wurde über die Anträge aus dem Antragsbereich M abgestimmt. ${ }^{27}$ Der Leitantrag „Freiheit, Gerechtigkeit und Solidarität in der Digitalen Gesellschaft" wurde als Beschluss Nr. 36 einstimmig angenommen.

Auf dem Parteitag selbst wurde das Online-Antragsverfahren explizit thematisiert, etwa in der Einbringungsrede von Björn Böhning:

„Dieser Antrag [...] ist ein gelebtes Zeichen der Öffnung der Partei. [...] An der Erstellung dieses Antrags waren sicher mehr Genossinnen und Genossen und auch Nichtmitglieder beteiligt, mehr als an jedem anderen Antrag, der Euch in diesem Antragsbuch vorliegt. [...] Dieser Antrag ist der demokratischste des Parteitags. “"28

Dieser normative Topos wurde in der Beratung des Antrags ein weiteres Mal aufgegriffen, von Matthias Groote, MdEP: „Mehr Demokratie wagen 2.0! Das Experiment ist gelungen!" 29

Eine im Rahmen des Parteitags durchgeführte Delegiertenbefragung ${ }^{30}$ zeigt wiederum, dass die Teilnehmer des Parteitags das besondere Antragsverfahren zuvor nicht wahrgenommen hatten und diesem keinen höheren Stellenwert als dem regulären Verfahren einräumten. 78,5 Prozent der Befragten haben erstmalig auf dem Parteitag von dem Online-Verfahren gehört. Die Mehrheit der Befragten spricht

27 Live-Ticker zum SPD Parteitag, www.spd.de/aktuelles/Parteitag_2011/21630/20111205_spd_par teitag_liveticker_zweitertag.html (Stand: 22.5.2012).

28 SPDvision, www.youtube.com/watch? $\mathrm{v}=2 \mathrm{kA} 2 \mathrm{X}$-hcdxU\&feature $=$ plcp (Stand: 15.5.2012).

29 SPD Parteitag, www.spd.de/aktuelles/Parteitag_2011/21652/20111205_antrag_netzpolitik_partei tag.html (Stand: 15.5.2012).

30 Im Rahmen einer durch das Institut für deutsches und europäisches Parteienrecht und Parteienforschung $(\mathrm{PRuF})$ der Heinrich-Heine-Universität Düsseldorf durchgeführten Delegiertenbefragung zur Organisationsreform der SPD auf dem Berliner Parteitag wurden auch Fragen zum SPD OnlineAntragsverfahren gestellt. Befragt wurden alle 525 auf dem Parteitag anwesenden Delegierten, inklusive der Mitglieder des Parteivorstands. Die Rücklaufquote lag bei 26 Prozent. 
einem in einem Online-Verfahren erstellten Antrag keine höhere Bedeutung zu. Nur acht Prozent geben an, dass die Aussage ,Ein online diskutierter Antrag hat für mich mehr Gewicht als andere Anträge“,,voll und ganz“" oder „,eher“ zutreffe (vgl. Tab. 3).

Tabelle 3: Bewertung des Online-Antragsverfahrens durch die Delegierten (Itemformulierung: ,Ein online diskutierter Antrag hat für mich mehr Gewicht als andere Anträge.")

\begin{tabular}{|c|l|c|c|}
\hline \multicolumn{2}{|c|}{} & Häufigkeit & Prozente \\
\hline \multirow{4}{*}{} & trifft voll und ganz zu & 4 & 2,9 \\
\cline { 2 - 4 } & trifft eher zu & 11 & 8,0 \\
\cline { 2 - 4 } & teils-teils & 16 & 11,7 \\
\cline { 2 - 4 } & trifft eher nicht zu & 56 & 40,9 \\
\cline { 2 - 4 } & trifft überhaupt nicht zu & 50 & 36,5 \\
\cline { 2 - 4 } & Gesamt & 137 & 100,0 \\
\hline
\end{tabular}

Quelle: PRuF-Delegiertenbefragung.

Positiver bewerten die befragten Delegierten allerdings die Möglichkeit, über Online-Verfahren auch Nichtmitglieder an Antragsdiskussionen zu beteiligen (vgl. Tab. 4); immerhin fast 40 Prozent begrüßen diese Option.

Tabelle 4: Bewertung der Einbindung von Nichtmitgliedern durch das Online-Verfahren (Itemformulierung: ,Ich finde es gut, dass über das Online-Verfahren auch Nichtmitglieder beteiligt werden können.")

\begin{tabular}{|l|l|c|c|}
\hline \multicolumn{2}{|c|}{} & Häufigkeit & Prozente \\
\hline \multirow{4}{*}{} & trifft voll und ganz zu & 16 & 11,8 \\
\cline { 2 - 4 } & trifft eher zu & 51 & 37,5 \\
\cline { 2 - 4 } & teils-teils & 37 & 27,2 \\
\cline { 2 - 4 } & trifft eher nicht zu & 18 & 13,2 \\
\cline { 2 - 4 } & trifft überhaupt nicht zu & 14 & 10,3 \\
\cline { 2 - 4 } & Gesamt & 136 & 100,0 \\
\hline
\end{tabular}

Quelle: PRuF-Delegiertenbefragung.

Insgesamt zeigen die Ergebnisse der Delegiertenbefragung, dass das positive Framing des Online-Antragsverfahrens durch eine differenziertere Wahrnehmung des Prozesses aufseiten der Parteitagsdelegierten empirisch ergänzt werden muss. 


\section{Diskussion}

$\mathrm{Zu}$ Beginn wurden auf der Grundlage theoretischer Erwägungen zwei Erwartungen an den Einsatz von internetbasierten Beteiligungsplattformen in Parteien abgeleitet, die nun auf der Grundlage der Ergebnisse der Fallstudie diskutiert werden können. Beide Thesen gingen davon aus, dass die Parteiführungen als rationale Akteure internetbasierte Partizipationsverfahren nur so einsetzen, dass sie die Kontrolle über Prozess und Ergebnis behalten können.

These 1: Während der Online-Phase wird aufgrund der Robustheit bestehender Willensbildungs- und Entscheidungsstrukturen die Parteiführung beim innerparteilichen Einsatz einer Partizipationsplattform die Prozesssteuerung behalten. In dem untersuchten Fall des SPD-Online-Antrags ist der Einsatz des Tools von der Parteiführung sowohl veranlasst als auch kontrolliert worden. Die Initiative für die Verwendung der Plattform ging von der Parteiführung aus. Die Adhocracy-Software wurde für die Verwendung angepasst, insbesondere durch die Installation eines Community Managements: In seiner Standardversion beruht Adhocracy auf dem Prinzip der „Selbstverwaltung "31 und verzichtet auf Formen der Moderation. Die Reichweite und Entscheidungsmacht des Community Managements zeigt sich insbesondere bei der Einordnung der Vorschläge auf der Plattform als thematisch passend oder unpassend. Des Weiteren wurden sowohl in der Auswahl des zu erstellenden Antragskapitels als auch in Form der Leitfragen, zu denen Vorschläge entwickelt werden konnten, thematische Vorgaben oder Engführungen seitens der Initiatoren gemacht. Die Beteiligung fand auf der Grundlage von „top down“-Vorgaben und unter einer „top down"-Prozessleitung statt. Außerdem wurde zwar das fertige Antragskapitel nach Abschluss der Online-Phase auf der Plattform eingestellt, eine abschließende Diskussion des Kapitels unter Einbeziehung der OnlineTeilnehmer wurde allerdings nicht ermöglicht. Die Online-Willensbildung und Offline-Entscheidungsfindung waren nicht unmittelbar, sondern nur über das ,,party in central office" verknüpft.

These 2: Nach der Online-Phase wird die Parteiführung die Steuerungskompetenz über die Verarbeitung des Ergebnisses nicht aus der Hand geben.

Auch bei der Ergebnisverarbeitung sind die Bemühungen der Parteiführung evident, die Kontrolle zu behalten. Im konkreten Fall wurde die Verarbeitung des Ergebnisses in drei Hinsichten gesteuert:

a) Die erste Einschränkung fand bereits im Vorfeld durch die Auswahl des Anwendungsgebiets des Verfahrens statt. Genutzt wurde dieses zur Erarbeitung von le-

31 www.adhocracy.de/_pages/about/uber-adhocracy (Stand: 15.5.2012). 
diglich einem Antragskapitel eines Leitantrages, der auf dem Parteitag nicht die zentrale Rolle gespielt hat, welcher vielmehr von europapolitischen Debatten sowie der geplanten Parteireform beherrscht wurde (Höll 2011). Der Antrag und das Verfahren spielten für die Delegierten des Parteitags eine nachgeordnete Rolle. Zudem beinhaltete der Leitantrag mit seinem online-generierten Kapitel keine konkreten Policy-Forderungen, sodass er keine unmittelbare Bindungskraft auf die Entscheidungsprozesse der Partei in den Parlamenten zeitigte.

b) Die zweite Einschränkung betrifft die Übernahme der Online-Vorschläge für den Leitantrag. Dieser Selektionsschritt folgte keinen erkennbaren Regeln außer dem Kriterium der thematischen Passgenauigkeit der Vorschläge für den Leitantrag: Vorschläge, die sich inhaltlich vom Thema des Leitantrags entfernt hatten, wurden nicht berücksichtigt. Welche der thematisch passenden Vorschläge übernommen wurden, lag in der Entscheidung der Plattformmoderatoren; es gab keinen objektiven Algorithmus (z. B. Anzahl der Unterstützungen, Kommentare oder Argumentationsstruktur), auf dessen Grundlage die Auswahl der Vorschläge vorgenommen wurde.

c) Die dritte Einschränkung bezieht sich auf die konkrete Verarbeitung der ausgewählten Vorschläge. Nur ein Drittel der online-entwickelten Beiträge wurde nahezu wörtlich in den Leitantrag eingebaut, ein Drittel wurde paraphrasiert und beim letzten Drittel lässt sich der Bezug zwischen Online-Beitrag und entsprechender Stelle im Leitantrag kaum ausmachen. Dabei ist diese Einschränkung auch in der Funktionalität begründet: Eine redaktionelle Bearbeitung der Vorschläge war erforderlich, allein um den formalen Ansprüchen an einen Antrag an den Bundesparteitag zu genügen.

Die beiden eingangs aufgestellten Erwartungen sind somit bestätigt worden: Die Parteiführung hat den Prozess als „top down“-Verfahren aufgezogen und die Steuerungsfähigkeit über den Verlauf und die Ergebnis(-verarbeitung) in der Hand behalten. Insofern wirkt das Partizipationsprojekt prima vista als „Spielwiese“, die auf dem Parteitag als demokratische Errungenschaft dargestellt wurde, die aber anscheinend in ihrer Durchführung an den innerparteilichen Hierarchien nichts geändert hat. 


\section{Fazit und Perspektiven}

Das Fallbeispiel bestätigt die Vermutung, dass (etablierte) Parteien internetbasierte Partizipationsplattformen nach der ,top down“-Logik in bestehende Strukturen ihrer Willensbildung einbinden. Dies spricht für die Valenz der Instrumentalisierungsthese.

Gleichwohl greift dieser Befund zu kurz; auch für die Partizipationsthese lassen sich innerhalb des ausgewählten Falles Hinweise dort finden, wo „bottom up“-Potenziale zumindest aufgeleuchtet sind. Zunächst betrifft dies die Möglichkeiten der Mitglieder, auf einen Leitantrag eines Parteitages einzuwirken. Laut SPD Organisationsstatut ${ }^{32}$ sind bislang auf Bundesebene Organisationsgliederungen (Ortsvereine, regionale Zusammenschlüsse), Arbeitsgemeinschaften und -kreise, Themenforen, nahestehende Organisationen (auf Bitte der Partei) und der Parteivorstand antragsberechtigt. Mithilfe des Online-Verfahrens konnten Mitglieder jenseits ihrer Zugehörigkeit zu einer regionalen oder funktionalen Parteigliederung an der Ausgestaltung eines Parteitagantrags mitwirken.

„Bottom up“-Potenziale sowie neue Linkage-Optionen liegen auch in der faktischen Öffnung des Antragsverfahrens für Nichtmitglieder. Die Teilnehmenden waren anonym, sodass nicht erkennbar war, wer Mitglied der SPD war und wer Sympathisant - oder möglicherweise sogar einer anderen Partei nahestand und -steht. ${ }^{33}$ Nichtmitglieder konnten wie die regulären Parteibuchinhaber gleichberechtigt im Prozess mitwirken, Vorschläge entwickeln, kommentieren und abstimmen. Die Trennlinie zwischen Mitglied und Nichtmitglied ist im Online-Verfahren aufgehoben, insbesondere weil das Prinzip der Anonymität beibehalten worden ist. Die Verwendung solcher Plattformen stärkt somit den „,friends“-Sektor der Parteien (Marschall 2012). Damit lässt sich dieses Verfahren in die Organisationsreform der SPD einordnen, die darauf zielte, die Partizipationsmöglichkeiten für die Mitglieder, aber auch für die Sympathisanten zu stärken.

Auf den zweiten Blick ist also das Maß an Mitsprache, das die Partei den Usern im Rahmen des Online-Antrags eingeräumt hat, durchaus bemerkenswert. Diesem Angebot der Partei stand freilich eine nur begrenzte Nachfrage seitens der Nutzer gegenüber. Die Partizipationsrate war vergleichsweise gering. Zudem wurden die kollaborativen Beteiligungstools nur sehr zurückhaltend verwendet. Die ungleiche

32 SPD-Organisationsstatut, www.spd.de/linkableblob/1852/data/Organisationsstatut.pdf (Stand: 7.3.2012).

$33 \mathrm{Ob}$ durch den Verzicht auf die Verpflichtung von Klarnamen die diskursive Qualität leidet oder gewinnt, ist umstritten. Eine Klarnamenpflicht kann sich außerdem auch auf die Beteiligungsbereitschaft auswirken (u. a. Baek/Wojcieszak/Delli Carpini 2012). 
Verteilung der User auf die Partizipationsformen entspricht jedoch der in Anlehnung an Nielsen (2006) gängigen Aufteilung von Nutzergruppen von Social Media-Angeboten in ,aktive“, „reaktive“ und „passive“ Nutzer (Michelis/Schildhauer 2012). Dabei spielen hier sicherlich auch die Charakteristika des Politikfelds und die vorsichtige Mobilisierung für den Online-Antrag eine Rolle. Schließlich: Da dieses Verfahren erstmalig in der SPD Verwendung gefunden hat, stieß das Angebot der Parteiführung (noch) nicht auf eine eingespielte Online-Partizipationskultur. Das könnte sich bei einer wiederholten Verwendung online-basierter Beteiligungsplattformen innerhalb der Partei merklich ändern.

Eine weitere Begrenzung der Aussagekraft der Befunde liegt in der Besonderheit des Falls. Es wurde der Einsatz eines solchen Tools bei einer traditionellen Mitgliederpartei thematisiert. Dass die Befunde bei der Piratenpartei und ihrer speziellen Form der Anwendung von internetbasierten Plattformen anders ausgefallen wären, ist evident. Dort wurde der Bundesparteitag in Offenbach, der fast zeitgleich zum SPD-Parteitag im Dezember 2011 stattfand, durchweg online-basiert vorbereitet und begleitet. So hielt beispielsweise das Wiki der Piratenpartei eine „Antragsfabrik" bereit, in der Anträge an den Bundesparteitag im Vorfeld diskutiert und bearbeitet werden konnten. ${ }^{34}$ Außerdem konnten Mitglieder, die bei den Piraten alle ausnahmslos antragsberechtigt sind, auf der „LiquidFeedback“-Plattform der Partei Antragsentwürfe mit anderen Mitgliedern abstimmen, um etwa Dopplungen von Anträgen zu vermeiden oder um bereits im Vorfeld innerhalb der Partei zu testen, wie aussichtsreich ein Antrag ist. ${ }^{35}$ Fertige Anträge konnten über ein Online-Antragsportal oder per Mail an die Antragskommission übermittelt werden. ${ }^{36}$

Die etablierten Parteien sind durch die Form der Einbindung solcher Plattformen insbesondere seitens der Piratenpartei unter Druck gesetzt worden (Bieber 2011 a; Hensel 2011). Aufgrund der bislang positiven Wahrnehmung internetvermittelter Partizipationsprojekte ist davon auszugehen, dass zukünftig vermehrt solche Verfahren im politischen Prozess eingesetzt werden.

Bei zunehmender innerparteilicher Verwendung werden die internetbasierten Beteiligungsplattformen eine Robustheit entfalten, die eine bloße Instrumentalisierung seitens der Parteizentralen unwahrscheinlich macht. Online-Tools bringen eine eigene „Medienlogik“ mit sich und entwickeln dabei eine eigene Dynamik, die sich einer umfassenden Steuerung durch die Parteiführung entzieht. So lassen sich zum

34 Antragsfabrik der Piratenpartei, wiki.piratenpartei.de/Bundesparteitag_2011.2/Antragsfabrik (Stand: 15.5.2012).

35 „LiquidFeedback“-Plattform der Piratenpartei, www.lqfb.piratenpartei.de/ (Stand: 15.5.2012).

36 Antragsportal der Piratenpartei, www.wiki.piratenpartei.de/Bundesparteitag_2011.2/Antragspor tal (Stand: 15.5.2012). 
Beispiel die Diskursverläufe und Themensetzungen nicht vollständig kontrollieren, was mitunter dazu führen kann, dass in Beteiligungsverfahren Positionen entwickelt werden, die der Programmatik einer Partei entgegenstehen. In diesem Prozess werden auch die Dienstleister, die derartige Tools (weiter)entwickeln und bereitstellen, zu Akteuren, die Einfluss ausüben können, weil sie letzten Endes über die machtressourcenrelevanten Algorithmen und Formate dieser „Medien“ mitentscheiden.

Angesichts der sich vermutlich ausweitenden Verfahren entsteht hier ein neues und relevantes Untersuchungsfeld für sozialwissenschaftliche Forschung; eine (vergleichende) Analyse weiterer Fälle kann Hinweise darauf geben, ob Instrumentalisierungs- oder Partizipationstendenzen zu verzeichnen sind und wie stark - kontextbedingt - die Eigenlogik des Formates ist. Ein Desiderat ist neben der Ausweitung von Inhaltsanalysen und Fallstudien eine genauere Untersuchung der Teilnehmerschaft an solchen Prozessen. Dabei gilt es insbesondere zu analysieren, inwieweit eine Einbindung bislang nicht aktiver Personen in politischen Prozessen oder nur die Vertiefung bestehender innerparteilicher Partizipationsungleichgewichte zu beobachten ist, das heißt sich möglicherweise an diesen Verfahren nur diejenigen beteiligen, die ohnehin zu den partizipationsstarken Personen (innerhalb der Parteien) gehören. Diese Problematik wird in der Kontroverse zwischen der Mobilisierungs-, Normalisierungs-, oder Abkehrthese diskutiert. ${ }^{37}$

Erst eine solche Analyse der Nutzerschaft ermöglicht Rückschlüsse auf die Fähigkeit dieser Tools, die Linkage-Probleme der Parteien zu lösen. Von ihrer Anlage her scheinen derartige Beteiligungsplattformen durchaus das Potenzial für eine dichtere Verkopplung von Parteielite und Parteibasis, aber auch für die Öffnung der Parteien in den Bereich der Nichtmitglieder zu bieten.

\section{Literatur}

Abelson, Julia/Gauvin, Francois-Pierre, 2006: Assessing the Impacts of Public Participation: Concepts, Evidence and Policy Implications, Canadian Policy Research Networks, Research Report 6, Ottawa.

Aichholzer, Georg/Westholm, Hilmar, 2009: Evaluating eParticipation Projects:

Practical Examples and Outline of an Evaluation Framework, in: European Journal of ePractice 7, 27-44.

Albers, Hagen, 2009: Onlinewahlkampf 2009, in: Aus Politik und Zeitgeschichte B 51, 33-38.

37 Vergleiche Grunwald u. a. 2006, Gibson/Lusoli/Ward 2005, Emmer/Vowe 2004 sowie Rheingold 1993. 
Alemann, Ulrich von, 1997: Parteien und Medien, in: Oscar W. Gabriel/Oskar Niedermayer/Richard Stöss (Hrsg.), Parteiendemokratie in Deutschland, Bonn, 478-494.

Alemann, Ulrich von, 2010: Das Parteiensystem der Bundesrepublik Deutschland. Grundwissen Politik, Wiesbaden (4. Aufl.).

Baek, Young Min/Wojcieszak, Magdalena/Delli Carpini, Michael X., 2012: Online Versus Face-to-Face Deliberation. Who? Why? What? With what effects?, in: New Media \& Society 14 (3), 1-21.

Bandelow, Nils C., 1999: Lernende Politik. Advocacy-Koalitionen und politischer Wandel am Beispiel der Gentechnologiepolitik, Berlin.

Beck, Klaus, 2010: Soziologie der Online-Kommunikation, in: Klaus Beck/Wolfgang Schweiger (Hrsg.), Handbuch Online-Kommunikation, Wiesbaden, 15-35. Beyme, Klaus von, 2000: Parteien im Wandel. Von den Volksparteien zu den professionalisierten Wählerparteien, Wiesbaden.

Bieber, Christoph, 1999: Politische Projekte im Internet. Online-Kommunikation und politische Öffentlichkeit, Frankfurt a. M.

Bieber, Christoph, 2011 a: Der Online-Wahlkampf im Superwahljahr 2009, in: Eva Johanna Schweitzer/Steffen Albrecht (Hrsg.), Das Internet im Wahlkampf. Analysen zur Bundestagswahl 2009, Wiesbaden, 69-98.

Bieber, Christoph, 2011 b: Aktuelle Formen der Politik(v)ermittlung im Internet, in: Peter Massing (Hrsg.), Politikvermittlung in der Demokratie, Politische Bildung 2, 50-67.

Bieber, Christoph, 2012: Die Piratenpartei als neue Akteurin im Parteiensystem, in: Aus Politik und Zeitgeschichte B 7, 27-33.

Biezen, Ingrid van/Mair, Peter/Poguntke, Thomas, 2012: Going, Going ... Gone? The Decline of Party Membership in Contemporary Europe, in: European Journal of Political Research 51 (1), 24-56.

Blatter, Joachim K./Janning, Frank/Wagemann, Claudius, 2007: Qualitative Politikanalyse. Eine Einführung in Forschungsansätze und Methoden. Wiesbaden.

Bücker, Teresa, 2011: Das Internet stärkt politisches Engagement - und somit die Parteien. Der Newsdesk im Willy-Brandt-Haus als Beispiel, in: Neue Gesellschaft, Frankfurter Hefte (11), 13-17.

Bukow, Sebastian, 2010: Die professionalisierte Parteiorganisation. Bedeutung und Selbstverständnis der Party Central Office, in: Uwe Jun/Benjamin Höhe (Hrsg.), Parteien als fragmentierte Organisationen, Opladen/Farmington Hills, 257-278. Busemann, Katrin/Gscheidle, Christoph, 2011: Web 2.0. Aktive Mitwirkung verbleibt auf niedrigem Niveau, in: Media Perspektiven (7-8), 360-369. 
Chadwick, Andrew, 2009: Web 2.0. New Challenges for the Study of E-Democracy in an Era of Informational Exuberance, in: Journal of Law and Politics for the Information Society 5 (1), 9-41.

Chadwick, Andrew, 2011: Explaining the Failure of an Online Citizen Engagement Initiative. The Role of Internal Institutional Variables, in: Journal of Information Technology \& Politics 8 (1), 21-40.

Christmann, Stefan/Melcher, Johannes/Hagenhoff, Svenja/Gissendanner, Scott Stock/Krumbein, Wolfgang, 2010: Web 2.0-Technologien in Meinungsbildungsprozessen von politischen Parteien, in: i-com. Zeitschrift für interaktive und kooperative Medien 9 (3), 21-27.

Detterbeck, Klaus, 2002: Der Wandel politischer Parteien in Westeuropa. Eine vergleichende Untersuchung von Organisationsstrukturen, politischer Rolle und Wettbewerbsverhalten von Großparteien in Dänemark, Deutschland, Großbritannien und der Schweiz, 1960-1999, Opladen.

Detterbeck, Klaus, 2005: Die strategische Bedeutung von Mitgliedern für moderne Parteien, in: Josef Schmid/Udo Zolleis (Hrsg.), Zwischen Anarchie und Strategie. Der Erfolg von Parteiorganisationen, Wiesbaden, 63-76.

Donges, Patrick, 2005: Medialisierung der Politik - Vorschlag einer Differenzierung, in: Friedrich Krotz/Patrick Rössler (Hrsg.), Mythen der Mediengesellschaft - The Media Society and its Myths, Konstanz, 321-339.

Donges, Patrick, 2008: Medialisierung politischer Organisationen. Parteien in der Mediengesellschaft, Wiesbaden.

Ebersbach, Anja/Glaser, Markus/Heigl, Richard, 2011: Social Web, Konstanz (2. Aufl.).

Emmer, Martin/Vowe, Gerhard, 2004: Mobilisierung durch das Internet? Ergebnisse einer empirischen Längsschnittuntersuchung zum Einfluss des Internets auf die politische Kommunikation der Bürger, in: Politische Vierteljahresschrift 45 (2), 191-212.

Emmer, Martin/Vowe, Gerhard/Wolling, Jens, 2011: Bürger online. Die Entwicklung der politischen Online-Kommunikation in Deutschland, Konstanz.

Gabriel, Oscar W./Falter, Jürgen W./Rattinger, Hans (Hrsg.), 2005: Wächst zusammen, was zusammen gehört? Stabilität und Wandel politischer Einstellungen im wiedervereinigten Deutschland, Baden-Baden.

Gehne, David/Spier, Tim (Hrsg.), 2010: Krise oder Wandel der Parteiendemokratie? Wiesbaden.

Gibson, Rachel K./Lusoli, Wainer/Ward, Stephen, 2005: Online Participation in the UK: Testing a 'Contextualised' Model of Internet Effects, British Journal of Politics and International Relations 7, 561-583. 
Gibson, Rachel K./Nixon, Paul/Ward, Stephen, 2003: Political Parties and the Internet. Net Gain?, New York.

Grunwald, Armin/Banse, Gerhard/Coenen, Christopher/Hennen, Leonhard, 2006: Netzöffentlichkeit und digitale Demokratie. Tendenzen politischer Kommunikation im Internet, Berlin.

Häder, Michael, 2010: Empirische Sozialforschung. Eine Einführung, Wiesbaden (2. Aufl.).

Heidar, Knut/Saglie, Jo, 2003: Predestined Parties? Organizational Change in Norwegian Political Parties, in: Party Politics 9 (2), 219-239.

Hensel, Alexander, 2011: Der Druck der Piraten auf die etablierten Parteien wächst, Cicero Online Magazin für politische Kultur, 6.12.2011, http://www.cicero.de/ blog/goettinger-demokratie-forschung/2011-12-06/der-druck-der-piraten-aufdie-etablierten-parteien (Stand: 22.5.2012).

Höll, Susanne, 2011: Geschlossen, nicht langweilig. Bilanz des SPD-Parteitags in Berlin, Süddeutsche Zeitung, www.sueddeutsche.de/politik/bilanz-des-spd-parteitags-in-berlin-geschlossen-aber-nicht-langweilig-1.1228258 (Stand: 22.5.2012).

Immerfall, Stefan, 1994: Parteienforschung in der Parteienkrise, in: Politische Vierteljahresschrift 35 (3), 480-492.

Jackson, Nigel A./Lilleker, Darren G., 2009: Building an Architecture of Participation? Political Parties and Web 2.0 in Britain, in: Journal of Information Technology \& Politics 6, 232-250.

Jun, Uwe, 2004: Der Wandel von Parteien in der Mediendemokratie. SPD und Labour Party im Vergleich, Frankfurt a. M.

Jun, Uwe, 2009: Parteien, Politik und Medien. Wandel der Politikvermittlung unter den Bedingungen der Mediendemokratie, in: Frank Marcinkowski/Barbara Pfetsch (Hrsg.), Politik in der Mediendemokratie (PVS Sonderheft 42), Wiesbaden, 270-295.

Katz, Richard S./Mair, Peter, 1993: The Evolution of Party Organizations in Europe. Three Faces of Party Organization, in: William Crotty (Hrsg.), Political Parties in a Changing Age (American Review of Politics 14), 593-617.

Katz, Richard S./Mair, Peter, 1995: Changing Models of Party Organization and Party Democracy. The Emergence of the Cartel Party, in: Party Politics 1 (1), 5-28.

Katz, Richard S./Mair, Peter, 2002: The Ascendancy of the Party in Public Office: Party Organizational Change in Twentieth-Century Democracies, in: Richard Gunther/Jose Ramon Montero/Juan Linz (Hrsg.), Political Parties: Old Concepts and New Challenges, Oxford, 113-35. 
Klein, Markus/Alemann, Ulrich von/Spier, Tim, 2011: Warum brauchen Parteien Mitglieder?, in: Tim Spier/Markus Klein/Ulrich von Alemann/Hanna Hoffmann/Annika Laux/Alexandra Nonnenmacher/Katharina Rohrbach (Hrsg.), Parteimitglieder in Deutschland, Wiesbaden, 19-29.

Klug, Astrid, 2012: Die Organisationsreform der SPD 2010/2011, in: Tobias Mörschel/Christian Krell (Hrsg.), Demokratie in Deutschland. Zustand, Herausforderungen, Perspektiven, Wiesbaden, 159-174.

Koole, Ruud, 1996: Cadre, Catch-all or Cartel? A Comment on the Notion of the Cartel Party, Party Politics 2 (4), 507-523.

Kubicek, Herbert/Westholm, Hilmar, 2010: Consensus Building 2010 by Blended Participation in a Local Planning Process: The Case of the Public Stadium Swimming Pool in Bremen, in: David Rios Insua/Simon French (Hrsg.), e-Democracy. A Group Decision and Negotiation Perspective, Dordrecht, 323-344.

Lamnek, Siegfried, 2005: Qualitative Sozialforschung, Weinheim/Basel (4. Aufl.). Leggewie, Claus/Bieber, Christoph, 2004: Interaktivität. Ein transdisziplinärer Schlüsselbegriff, Frankfurt a. M./New York.

Leibholz, Gerhard, 1967: Strukturprobleme der modernen Demokratie, Karlsruhe (3. Aufl.).

Lewitzki, Markus, 2011: Das Internet in Parteiform: Wie segelt die Piratenpartei?, regierungsforschung.de, Student Paper, Universität Duisburg-Essen, http://regi erungsforschung.de/data/070111_regierungsforschung.de_lewitzki_piraten.pdf (Stand: 23.5.2012).

Lilleker, Darren G./Pack, Mark/Jackson, Nigel, 2010: Political Parties and Web 2.0: The Liberal Democrat Perspective, in: Politics 30 (2), 105-112.

Linden, Markus/Thaa, Winfried (Hrsg.), 2011: Krise und Reform der repräsentativen Demokratie, Baden-Baden.

Lübcke, Maren/Lührs, Rolf, 2008: Haushaltsplanung 2.0. Symbolische Politik oder Mitbestimmung? in: Franz-Reinhard Habbel/Andreas Huber (Hrsg.), Web 2.0 für Kommunen und Kommunalpolitik. Neue Formen der Zusammenarbeit von Politik, Wirtschaft, Verwaltung und Bürgern, Boizenburg, 71-77.

Märker, Oliver/Wehner, Josef, 2008: Haushaltsplanung 2.0. E-Partizipation über Bürgerhaushalte, in: Franz-Reinhard Habbel/Andreas Huber (Hrsg.), Web 2.0 für Kommunen und Kommunalpolitik. Neue Formen der Zusammenarbeit von Politik, Wirtschaft, Verwaltung und Bürgern, Boizenburg, 63-68.

Marschall, Stefan, 2012: „Mitgliederpartei 2.0“- Chancen und Grenzen virtueller Parteimitgliedschaft, in: Ulrich von Alemann/Martin Morlok/Tim Spier (Hrsg.), Parteien ohne Mitglieder?, Baden-Baden (i. E.). 
Mazzoleni, Gianpietro/Schulz, Winfried, 1999: „Mediatization“ of Politics: A Challenge for Democracy? in: Political Communication 16 (3), 247-262.

Meckel, Miriam, 2008: Aus Vielen wird das Eins - wie Web 2.0 unsere Kommunikation verändert, in: Aus Politik und Zeitgeschichte B 39, 17-23.

Michelis, Daniel/Schildhauer, Thomas (Hrsg.), 2012: Social Media Handbuch. Theorien, Methoden, Modelle und Praxis, Baden-Baden (2. Aufl.).

Müller, Wolfgang C./Strøm, Kaare, 1999: Political Parties and Hard Choices. in: Wolfgang C. Müller/Kaare Strøm (Hrsg.), Policy, Office, or Votes? How Political Parties in Western Europe Make Hard Decisions, Cambridge, 1-35.

Niedermayer, Oskar, 2010: Erfolgsbedingungen neuer Parteien im Parteiensystem am Beispiel der Piratenpartei Deutschland, in: Zeitschrift für Parlamentsfragen (4), 838-854.

Nielsen, Jacob, 2006: Participation Inequality: Encouraging More Users to Contribute, http://www.useit.com/alertbox/participation_inequality.html (Stand: 21.5.2012).

Poguntke, Thomas, 2000: Parteiorganisation im Wandel. Gesellschaftliche Verankerung und organisatorische Anpassung im europäischen Vergleich, Wiesbaden.

Pöttgen, Andy, 2011: Die Partei auf digitalen Wegen, in: Vorwärts, 9.8.2011, www.vorwaerts.de/artikel_archiv/28278/die-partei-auf-digitalen-wegen.html (Stand: 21.5.2012).

Rheingold, Howard, 1993: The Virtual Community. Homesteading on the Electronic Frontier, Reading.

Roleff, Daniel, 2012: Digitale Politik und Partizipation: Möglichkeiten und Grenzen, in: Aus Politik und Zeitgeschichte B 7, 14-20.

Rössler, Patrick, 2010: Inhaltsanalyse, Konstanz (2. Aufl.).

Sarcinelli, Ulrich, 2004: Zur Unterschätzung der Eigenlogik des Politischen. Plädoyer für eine Rekontextualisierung der politischen Kommunikationsforschung, in: Kurt Imhof/Roger Blum/Heinz Bonfadelli/Otfried Jarren (Hrsg.), Mediengesellschaft (Reihe Mediensymposium Luzern, Bd. 8), Wiesbaden, 400-409.

Sarcinelli, Ulrich, 2011: Medien und Demokratie. Demokratie in Deutschland 2011

- Ein Report der Friedrich-Ebert-Stiftung, http://www.demokratie-deutschland -2011.de/common/pdf/Medien_und_Demokratie.pdf (Stand: 23.5.2012).

Sarcinelli, Ulrich/Wissel, Manfred, 1996: „Internetisierung“"von Öffentlichkeit und Demokratie. Trends, Chancen und Probleme für Politikvermittlung und politische Bildung im Online-Zeitalter, in: Friedrich-Ebert-Stiftung (Hrsg.), Jahrbuch für politische Bildung 1996 - Medien, Politik und politische Bildung, Bonn, 31-44. 
Schade, Edzard, 2004: Indikatoren für die Medialisierungsforschung. Konzepte von Wirklichkeitskonstruktion als Bausteine der Mediengesellschaft, in: Kurt Imhof/ Roger Blum/Heinz Bonfadelli/Otfried Jarren (Hrsg.), Mediengesellschaft (Reihe Mediensymposium Luzern, Bd. 8), Wiesbaden, 114-138.

Schmidt, Jan, 2011: Das neue Netz. Merkmale, Praktiken und Folgen des Web 2.0, Konstanz (2. Aufl.).

Schulz, Winfried, 2011: Politische Kommunikation. Theoretische Ansätze und Ergebnisse empirischer Forschung, Wiesbaden (3. Aufl.).

Spier, Tim, 2011: Wie aktiv sind die Mitglieder der Parteien?, in: Tim Spier/Markus Klein/Ulrich von Alemann/Hanna Hoffmann/Annika Laux/Alexandra Nonnenmacher/Katharina Rohrbach (Hrsg.), Parteimitglieder in Deutschland, Wiesbaden, 97-119.

Stromer-Galley, Jennifer, 2000: Online Interaction and Why Candidates Avoid It, in: Journal of Communication 50 (4), 111-132.

Tedesco, John C., 2007: Examining Internet Interactivity Effects on Young Adult Political Information Efficacy, in: American Behavioral Scientist 50 (9), 1183-1194.

Vogelmann, Frieder, 2012: Der Traum der Transparenz. Neue alte Betriebssysteme, in: Christoph Bieber/Claus Leggewie (Hrsg.), Unter Piraten, Bielefeld, 101-112. Ward, Stephen/Gibson, Rachel K., 2009: European Political Organizations and the Internet: Mobilization, Participation, and Change, in: Andrew Chadwick/Philip N. Howard (Hrsg.), Routledge Handbook of Internet Politics, London, 25-39.

Weiß, Ralph, 2009: Politisch-kommunikative Milieus. Notwendigkeit und Nutzen einer milieutheoretischen Analyse politischer Kommunikation, in: Medien und Kommunikationswissenschaft 57 (1), 3-22.

Wiesendahl, Elmar, 2002: Parteienkommunikation parochial - Hindernisse beim Übergang in das Online-Parteienzeitalter, in: Ulrich von Alemann/Stefan Marschall (Hrsg.), Parteien in der Mediendemokratie, Wiesbaden, 364-389.

Wiesendahl, Elmar, 2009: Die Mitgliederparteien zwischen Unmodernität und wieder entdecktem Nutzen, in: Uwe Jun/Oscar Niedermayer/Elmar Wiesendahl (Hrsg.), Zukunft der Mitgliederpartei, Opladen/Framington Hills, 31-52.

Wiesendahl, Elmar, 2010: Der Organisationswandel politischer Parteien. Organisations- und wandlungstheoretische Grundlagen, in: Uwe Jun/Benjamin Höhne (Hrsg.), Parteien als fragmentierte Organisationen, Opladen/Farmington Hills, 35-61.

Wiesendahl, Elmar, 2012: Partizipation und Engagementbereitschaft in Parteien, in:

Tobias Mörschel/Christian Krell (Hrsg.), Demokratie in Deutschland. Zustand, Herausforderungen, Perspektiven, Wiesbaden, 121-157. 
Wiesendahl, Elmar/Jun, Uwe/Niedermayer, Oskar, 2009: Die Zukunft der Mitgliederpartei auf dem Prüfstand, in: Elmar Wiesendahl/Uwe Jun/Oskar Niedermayer (Hrsg.), Zukunft der Mitgliederpartei, Opladen/Framington Hills, 9-30.

Wölk, Michaela/Oertel, Britta/Oppermann, Jan/Scheermesser, Mandy, 2008: Online-Dialogangebote von Bundestag und Bundesregierung. Quantiative und qualitative Analysen, Zukunftsstudien B 33, Frankfurt a. M. u. a.

Korrespondenzanschrift:

Katharina Hanel, M.A.

Heinrich-Heine-Universität Düsseldorf

Institut für Sozialwissenschaften, Politikwissenschaft II

Universitätsstraße 1

40225 Düsseldorf

E-Mail: katharina.hanel@uni-duesseldorf.de

Prof. Dr. Stefan Marschall

Heinrich-Heine-Universität Düsseldorf

Institut für Sozialwissenschaften, Politikwissenschaft II

Universitätsstraße 1

40225 Düsseldorf

E-Mail: stefan.marschall@uni-duesseldorf.de 